\title{
PECTUS EXCAVATUM / PECTUS CARINATUM: TRATAMENTO CIRÚRGICO
}

\author{
PECTUS EXCAVATUM / PECTUS CARINATUM: SURGICAL TREATMENT
}

\author{
Marlos de Souza Coelho, TCBC-PR ${ }^{1}$ \\ Wilson de Souza Stori Júnior ${ }^{2}$ \\ Lauro Del Valle Pizarro, TCBC- PR ${ }^{3}$ \\ Sérgio Augusto Zanin ${ }^{4}$ \\ José Lino Gonçalves ${ }^{4}$ \\ Nelson Bergonse Neto 5
}

\begin{abstract}
RESUMO: Objetivo: Apresentar os resultados obtidos com técnica única para tratamento do Pectus Excavatum e Pectus Carinatum. Método: De 1976 a 2000 foram operados, 183 portadores de Deformidades da Parede Torácica Anterior sendo 98 Pectus Carinatum (70 P. Carinatum Simétrico, 18 P. Carinatum Lateral a Direita e 10 P. Carinatum Lateral a esquerda), 62 Pectus Excavatum (57 P. Excavatum Simétrico, 4 P. Excavatum Lateral a Direita e um P. Excavatum Lateral a Esquerda), 17 Pectus Carinatum Superior, um Pectus Combinado, quatro Protusões Costais Inferiores e uma Depressão Costal. A indicação foi exclusivamente estética em 182 (99,4\%) dos pacientes. Foi utilizada técnica única para Pectus Carinatum e Pectus Excavatum: incisão transversal inframamária; ressecção subpericondral de todas as cartilagens envolvidas na deformidade; dissecção retroesternal mínima; osteotomia esternal anterior, fixação da osteotomia esternal com fios de aço; utilização da placa retroesternal em casos selecionados de Pectus Excavatum; pregueamento dos feixes pericondriais para dar maior rigidez a parede torácica e auxílio na manutenção do esterno na sua posição; drenagem do tecido celular subcutâneo e do plano submuscular, sutura intradérmica da pele. Resultados: Bom ou excelente em $175(95,6 \%)$ dos pacientes. Complicações ocorreram em $14(7,6 \%)$ pacientes: oito casos (4,5\%) de seroma; um $(0,5 \%)$ hematoma de parede; dois $(1,0 \%)$ caso de dor torácica intensa no pós-operatório; um $(1,0 \%)$ caso de deiscência parcial da sutura da pele e dois casos $(1,0 \%)$ de cicatriz hipertrófica que foram tratados com ressecção e betaterapia. Conclusão: Pelos resultados estéticos alcançados, a esternocondroplastia apresentada está indicada para correção de Pectus Excavatum/Carinatum.
\end{abstract}

Descritores: Tórax em funil; Anormalidades; Procedimentos cirúrgicos operatórios.

\section{INTRODUÇÃO}

Pediatras, ortopedistas, cirurgiões torácicos, cirurgiões pediátricos; cirurgiões plásticos, de modo geral, têm dificuldade em reconhecer os tipos de deformidades torácicas e em orientar ou conduzir o seu tratamento. O desconhecimento, a pouca experiência da maioria dos serviços de ortopedia e cirurgia torácica e a baixa incidência desta entidade contribui para esta dificuldade.

1. Mestre e Doutor em Cirurgia ; Professor Adjunto de Cirurgia da Pontifícia Universidade Católica do Paraná; Chefe do Serviço de Cirurgia Torácica e Endoscopia Respiratória do Hospital Universitário Cajuru da Pontifícia Universidade Católica do Paraná (HUC - PUC - PR) ; Titular da Sociedade Brasileira de Cirurgia Torácica.

2. Médico Assistente e Preceptor da Residência Médica do Serviço de Cirurgia Torácica e Endoscopia Respiratória do HUC-PUC-PR

3. Chefe do Serviço de Cirurgia Torácica da Santa Casa de Misericórdia de Curitiba; Titular da Sociedade Brasileira de Cirurgia Torácica.

4. Ex-Médico Residente em Cirurgia Torácica do Hospital Universitário Cajuru PUC - PR

5. Médico Residente do $3^{\circ}$ ano em Cirurgia Torácica do Hospital Universitário Cajuru PUC - PR de Curitiba- Pontifícia Universidade Católica do Paraná. 
É fundamental o conhecimento da sua classificação para o tratamento. Pectus Excavatum, conhecido entre os leigos como "peito de sapateiro", é uma deformidade em depressão do esterno e cartilagens costais inferiores, eventualmente acompanhada de deformidade da extremidade das costelas na sua articulação com as cartilagens costais. Muito embora Hipócrates citado por Ravitch ${ }^{1} \mathrm{e}$ Shamberger ${ }^{2}$ já tivesse feito referência à "deformidades do tórax", atribui-se a Bauhinus apud 3 o primeiro relato.

Existem várias teorias etiopatogênicas, principalmente relacionando o diafragma na gênese do Pectus Excavatum ${ }^{4,5}$. A teoria mais aceita, entretanto, utilizada inclusive como base para o tratamento cirúrgico, é de que haveria crescimento exagerado das cartilagens costais, que empurraria o esterno para trás, deprimindo-o. Permanece inexplicável o mecanismo deste hipercrescimento condral ${ }^{1,4,5}$

O desvio do coração para a esquerda nos portadores de Pectus Excavatum, observado em radiografia do tórax, ecocardiografia e tomografia axial computadorizada do tórax, levou à teoria que tal fato seria causa e não conseqüência: faltaria o suporte do coração para o esterno e/ou a pressão negativa intratorácica atrairia o esterno para dentro ${ }^{6}$.

A incidência varia de $0,58 / 1000$ a 3/1000 1,7,8 (Tabela 1).

História familiar da deformidade torácica ocorre de $23 \%$ a $41 \%$ e há predominância absoluta do sexo masculino para o feminino, de quatro a nove para um ${ }^{1,5-12}$. $\mathrm{O}$ pectus carinatum não tem merecido, por parte dos clínicos, pediatras, ortopedistas e cirurgiões pediátricos, o mesmo interesse que o Pectus Excavatum. A incidência, varia de $0,6 / 1000$ a $0,97 / 1000^{4,6}$ (Tabela 1 ).

Tabela 1 - Deformidades Torácicas: Incidência em 106.709 Escolares em Curitiba - 1982.

\begin{tabular}{lccc}
\hline Deformidades Torácicas & Número & Percentual & Incidência (em 1000) \\
\hline Pectus Carinatum Inferior & 104 & 46,64 & 0,97 \\
Pectus Excavatum & 62 & 27,80 & 0,58 \\
Pectus Carinatum Superior & 22 & 9,86 & 0,20 \\
Protusão condro costal inf. & 23 & 10,31 & 0,21 \\
"Tórax Chato" & 02 & 0,90 & 0,01 \\
Depressão condro costal & 06 & 2,69 & 0,05 \\
Síndrome de Poland & 04 & 1,79 & 0,03 \\
\hline Total & 223 & 100,00 & 2,05 \\
\hline
\end{tabular}

O pectus Carinatum tem sido classificado basicamente em dois tipos:

1. Pectus Carinatum inferior ${ }^{4,10,11}$ (Chickenbreast, Pigeon breast, Peito de Pombo). É a deformidade clássica, constituída pela proeminência do esterno, principalmente na sua porção média e inferior, e acompanhada quase sempre, de depressão costal bilateral inferior, causada pelo encurvamento para baixo das cartilagens costais e das extremidades das costelas. A radiografia do tórax em perfil mostra protusão do esterno para diante com espaços das cartilagens de conjugação esternais evidentes. A tomografia linear e a computadorizada do esterno mostra que a articulação (sincondrose) manúbrio-gladiolar é normal, bem como os núcleos de ossificação esternais. Esta deformidade esta presente no nascimento, de forma discreta, em pequeno número de pacientes, tornando-se mais visível na puberdade quando se acentua o seu crescimento. Nós denominamos por acharmos que tem a mesma etiopatogenia, de Pectus Carinatum Simétrico ou Clássico e Pectus Carinatum Lateral ou Assimétrico. No caso de Pectus Carinatum Assimétrico a tomografia computadorizada do tórax evidencia a obliqüidade do esterno em relação ao eixo do corpo, importante no planejamento cirúrgico.

2. Pectus Carinatum Superior ${ }^{10}$ (Pouter Pigeon, Pectus, Pectus Misto, Protusão Condro-manubrial com Depressão Condro-gladiolar ou Síndrome de Currarino Silvermam) ${ }^{12}$. É constituído de protusão superior do manúbrio e proximal do corpo ou gladíolo e depressão aguda na porção inferior do esterno, na maioria das vezes, pseudodepressão inferior. Para alguns seria forma mista de Pectus Excavatum / Carinatum. 
A radiografia do tórax em perfil mostra $\mathrm{o}$ esterno arciforme, às vezes em forma de "S" como osso único, menor que o esperado, com união completa entre o corpo e o manúbrio esternal. Tomografia do esterno e tomografia computadorizada do tórax mostra ausência da articulação manúbrio-gladiolar com obliteração das placas cartilaginosas de crescimento esternais e ossificação de todos os núcleos de ossificações esternais ${ }^{4,10,12}$.

Outras formas bizarras podem ocorrer, que a nosso ver são variantes das descritas acima. A protusão costal superior ou inferior, uni ou bilateral, são a nosso ver, alterações isoladas das cartilagens costais.

O Pectus Carinatum Clássico é percebido ao nascimento em número menor que o Pectus Excavatum, motivo pelo qual acredita-se que a sua ocorrência seja mais adquirida que congênita. $\mathrm{Na}$ maioria das vezes é percebido por volta dos 10 anos de idade. Currarino e Silverman ${ }^{12}$ demonstraram que a obliteração prematura das suturas esternais e dos núcleos de ossificação esternais é característica do Pectus Pouter Pigeon.

Ravitch ${ }^{13}$ e Haller ${ }^{14}$ consideram que a deformidade seria causada pelo crescimento exagerado das cartilagens envolvidas na deformidade. Tal hipercrescimento se bilateral e simétrico empurraria o esterno para diante (Pectus Carinatum) ou para trás (Pectus Escavatum). Caso o crescimento fosse unilateral ou assimétrico, a protusão seria unilateral ou predominante unilateral. Não é explicado o que levaria a este crescimento.porém esta é a teoria mais aceita, inclusive como base para o tratamento cirúrgico. A nossa observação é que o Pectus Carinatum se acentua na adolescência ${ }^{10}$.

Apesar da deformidade ser evidente, os médicos, principalmente os pediatras, afirmam que "não é doença", levando complexo e insegurança ao paciente e familiares.

Os ortopedistas, de modo geral, indicam tratamento baseado na compressão do tórax no sentido de moldar a parede torácica.

Por outro lado, os cirurgiões, principalmente os torácicos, acreditam que a cirurgia é a única forma de tratamento, inclusive para as formas discretas.

A maioria dos autores indica o tratamento cirúrgico desde que a deformidade seja realmente evidente ou grotesca. A indicação é de ordem estética na maioria das vezes e para prevenção e tratamento de problemas psicológicos e posturais ${ }^{2,10}$.
O objetivo deste trabalho é apresentar técnica para correção de Pectus Carinatum e Pectus Excavatum bem como os resultados deste tratamento.

\section{MÉTODO}

De 1976 a 2000 foram operados, no serviço de Cirurgia Torácica e Endoscopia Respiratória do Hospital Universitário Cajuru da Pontifícia Universidade Católica do Paraná, 183 portadores de deformidades na parede torácica anterior sendo 98 Pectus Carinatum (70 Pectus Carinatum Simétrico, 18 Pectus Carinatum Lateral a direita e 10 Pectus Carinatum Lateral a Esquerda), 62 Pectus Excavatum (57 Pectus Excavatum Simétrico,quatro Pectus Excavatum Lateral a Direita e um Pectus Excavatum Lateral a Esquerda), 18 Pectus Misto (17 Pectus Carinatum Superior e um Pectus Combinado), quatro Protusões Costais Inferiores e uma Depressão Costal (Tabela 2).

A idade variou dos cinco aos 40 anos, sendo que $155(84,6 \%)$ dos pacientes tinham idade abaixo dos vinte anos (Tabela 3$)$. Cento e cinqüenta $(81,9 \%)$ pacientes eram do sexo masculino e $33(18,1 \%)$ do sexo feminino.

A indicação foi exclusivamente estética em $182(99,4 \%)$ dos pacientes. Apenas um $(0,5 \%)$ paciente teve indicação funcional pulmonar. Sessenta e um $(98,3 \%)$ dos pacientes portadores de Pectus Excavatum foram submetidos a provas de função pulmonar que se revelaram normais. Um paciente não pode realizar a espirometria devido à deficiência mental. Um paciente de 22 anos com Pectus Carinatum com aspecto grosseiro apresentou comunicação interatrial associada à drenagem anômala das veias pulmonares esquerdas tendo sido submetido à cirurgia cardíaca e seis meses após, a correção da deformidade torácica.

Vinte e sete $(38,5 \%)$ dos pacientes portadores de Pectus Carinatum apresentavam asma brônquica ou bronquite associada.

\section{Indicação cirúrgica / não cirúrgica}

a) Deformidade evidente ou grotesca e o paciente ou familiares a deseje: indicação cirúrgica.

b) Idade para a cirurgia: qualquer idade, inclusive adulta. Nós preferimos operar após os dez anos. Operamos entre três e cinco anos quando a deformidade é muito acentuada. 
Tabela 2 - Deformidades Torácicas - Esternocondroplastia.

\begin{tabular}{lcc}
\hline Deformidades Torácicas & Número & Percentual \\
\hline Pectus Carinatum Inferior & $\mathbf{9 8}$ & $\mathbf{5 3 , 5}$ \\
· Simétrico & 70 & 37,7 \\
· Lateral Direito & 18 & 9,8 \\
· Lateral Esquerdo & 10 & 5,4 \\
Pectus Excavatum & $\mathbf{6 2}$ & $\mathbf{3 3 , 8}$ \\
· Simétrico & $57^{*}$ & 31,1 \\
· Lateral Direito & 04 & 2,1 \\
· Lateral Esquerdo & 01 & 0,5 \\
Pectus Carinatum Superior & $\mathbf{1 7}$ & $\mathbf{9 , 2}$ \\
Pectus Mixto/Combinado & $\mathbf{0 1}$ & $\mathbf{0 , 5}$ \\
Protusão Costal Inferior & $\mathbf{0 4}$ & $\mathbf{2 , 1}$ \\
Depressão Costal Inferior & $\mathbf{0 1}$ & $\mathbf{0 , 5}$ \\
\hline Total & $\mathbf{1 8 3}$ & $\mathbf{1 0 0 , 0}$ \\
\hline
\end{tabular}

* 1 Pectus Excavatum Recidivado.

Tabela 3 - Idade-Deformidades Torácicas Esternocondroplastia.

\begin{tabular}{lcc}
\hline Idade (anos) & Número & Percentual \\
\hline $00-05$ & 11 & 6,0 \\
$06-10$ & 18 & 9,8 \\
$11-15$ & 85 & 46,4 \\
$16-20$ & 41 & 22,4 \\
$21-25$ & 18 & 9,8 \\
$26-30$ & 05 & 2,7 \\
$31-35$ & 03 & 1,6 \\
$36-40$ & 02 & 1,0 \\
\hline Total & 183 & 100,0 \\
\hline
\end{tabular}

c) Deformidades discretas: observação e natação.

d) Pectus Carinatum em pacientes em fase de crescimento e deformidade progressiva ou acentuada foram encaminhadas para tratamento ortopédico.

\section{Técnica cirúrgica para pectus excavatum e pectus carinatum}

Incisão submamária bilateral, ocasionalmente longitudinal; dissecção da pele e tecido celular subcutâneo (TCS) a cautério, sendo que acima até o limite superior da deformidade cartilagínea e abaixo até o limite inferior da deformidade condrocostal. Os músculos peitorais são liberados na linha mediana por incisão longitudinal e dissecados até as articulações condrocostais, bilateralmente. Inferiormente, são desinseridos os músculos retos abdominais e oblíquos externos e internos para expor a porção inferior do esterno e das cartilagens. Incisa-se o pericôndrio a cautério e realiza-se a ressecção subpericondral das cartilagens envolvidas no defeito com descoladores apropriados. Habitualmente resseca-se da terceira a sétima cartilagem costal. A ressecção é sempre bilateral, mesmo no Pectus Assimétrico, pois o lado no qual as cartilagens permanecem, estas empurrarão o esterno para diante no Pectus Carinatum, e para trás, no Pectus Excavatum promovendo a recidiva da deformidade. Libera-se o processo xifóide, que quando muito angulado, é ressecado. No Pectus Carinatum Clássico, na maioria das vezes o esterno já toma a posição normal, não sendo necessária osteotomia. No Pectus Carinatum no qual o esterno não adquire posição correta, no Pectus Mixto e no Pectus Excavatum, libera-se o esterno do tecido frouxo mediastinal, do pericárdio e das pleuras bilateralmente com cuidado para não perfura-la (Figuras 1,2, 3 e 4). Para se corrigir, adequadamente, o defeito é necessário, ás vezes, que a osteotomia atinja as duas corticais. A fixação da osteotomia é feita com dois fios de Aciflex 5. Em 25 pacientes portadores de Pectus Excavatum acentuado, para "hipercorreção" do defeito e proporcionar melhor contorno a parede torácica anterior, utilizamos placa metálica retroesternal, transversal, fixada bilateralmente nas costelas com fio Aciflex 4. Nesse ponto regulariza-se as extremidades ósseas e condrais para que não façam saliência na pele e o contorno do tórax seja o mais perfeito possível. 
Pregueia-se o excesso de pericôndrio com polivicryl 0 de modo a dar estabilidade a parede torácica anterior e auxiliar na manutenção do esterno na posição. Os músculos peitorais são suturados entre si na linha mediana, e os músculos abdominais são suturados a margem inferior da musculatura peitoral. Aproximase o TCS com polivicryl 000 incolor e procede-se a sutura intradérmica da pele. Coloca-se um dreno tipo Suctor no tecido celular subcutâneo e um no plano submuscular. Os drenos são retirados em 24 - 48 horas e o paciente tem alta no quarto ou quinto dia de pós-operatório.

Os pacientes tem alta hospitalar no quarto dia pós operatório e são revistos em quinze dias, um mês, 6 meses e anualmente até completar cinco anos (Figura 5).
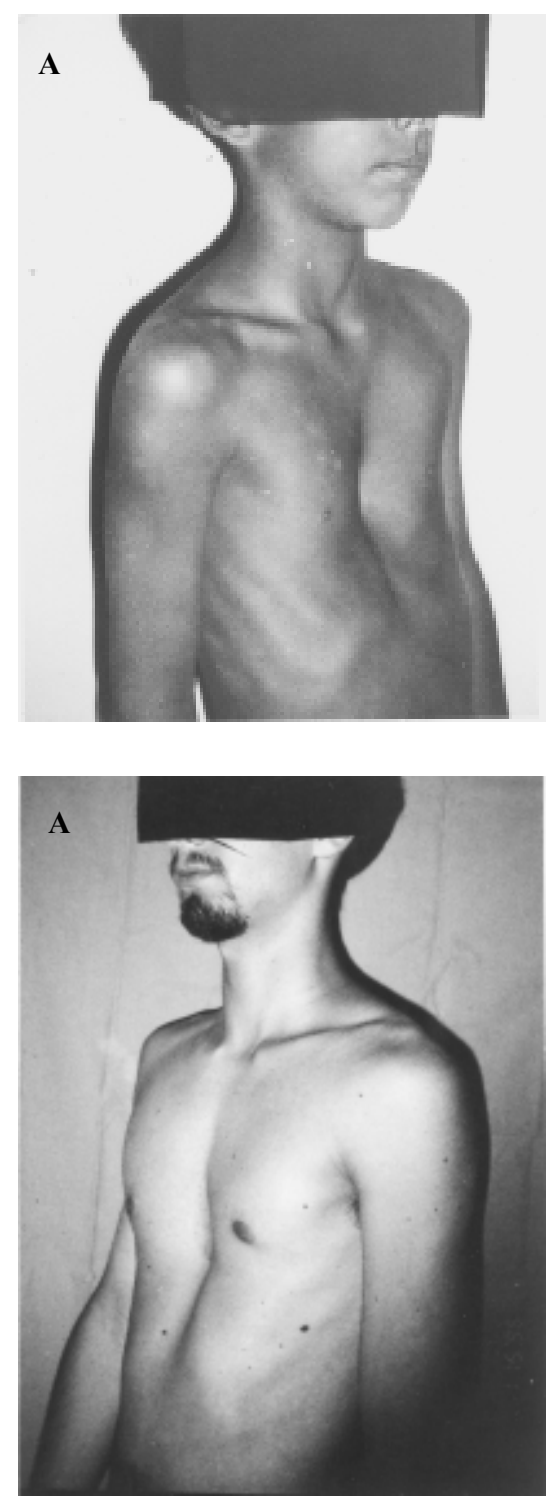

Os pacientes têm a recomendação de não praticar atividades físicas por três meses após os quais podem levar vida normal.

As protusões condrocostais são tratadas com incisão arciforme sobre as cartilagens envolvidas e ressecção subpericondral.

Uma paciente portadora de Pectus Carinatum Assimétrico apresentava hipoplasia mamária associada. Uma paciente com Pectus Carinatum severo foi submetida concomitantemente a mastoplastia redutora.

A(s) osteotomia(s) é(são) feita(s) sempre anteriormente, com escopo e martelo. A proteção das estruturas mediastinais é feita com valvas, variando a inclinação da(s) osteotomia(s) conforme a necessidade (Figura 6).
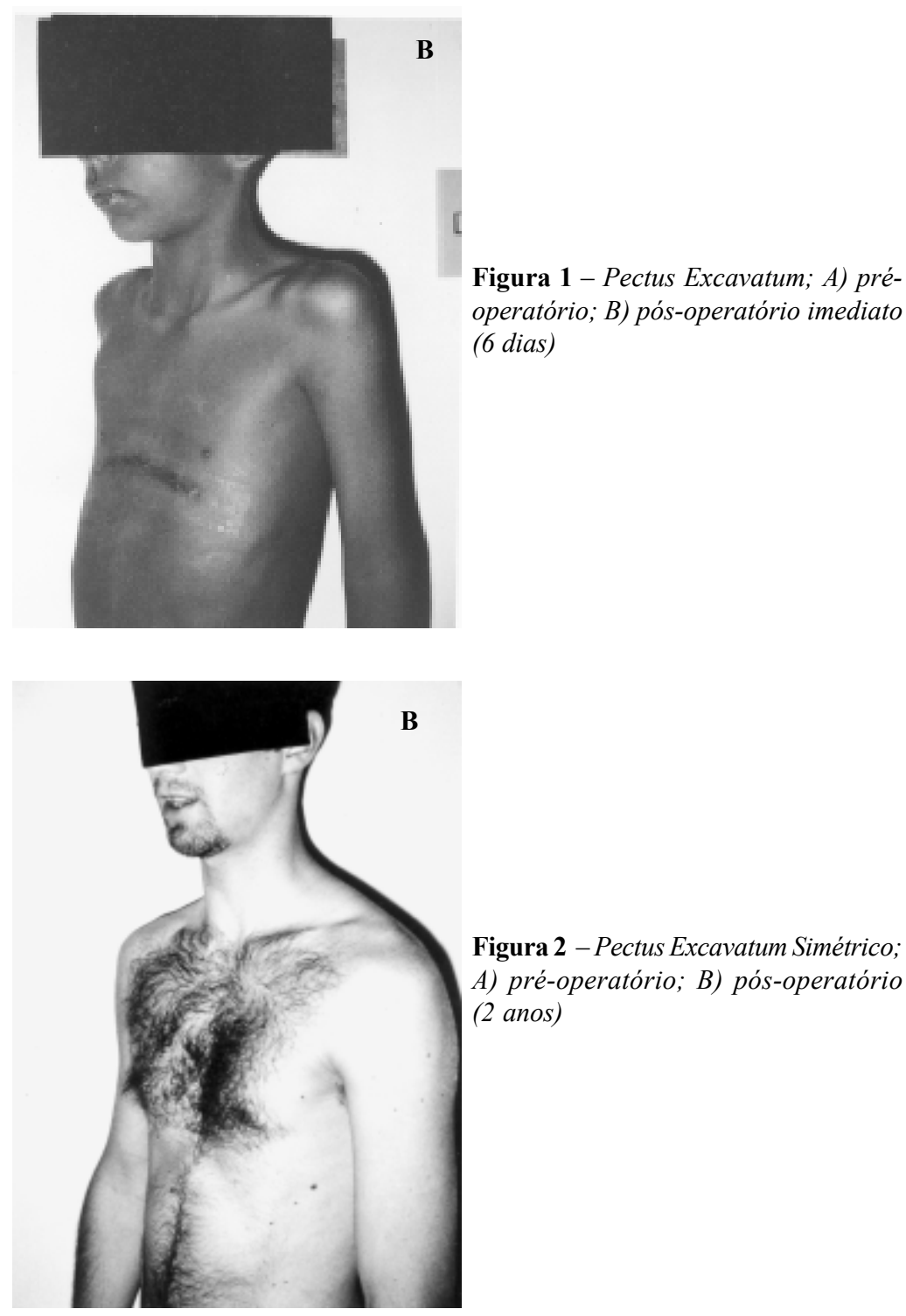

Figura 2 -Pectus Excavatum Simétrico; A) pré-operatório; B) pós-operatório (2 anos) 

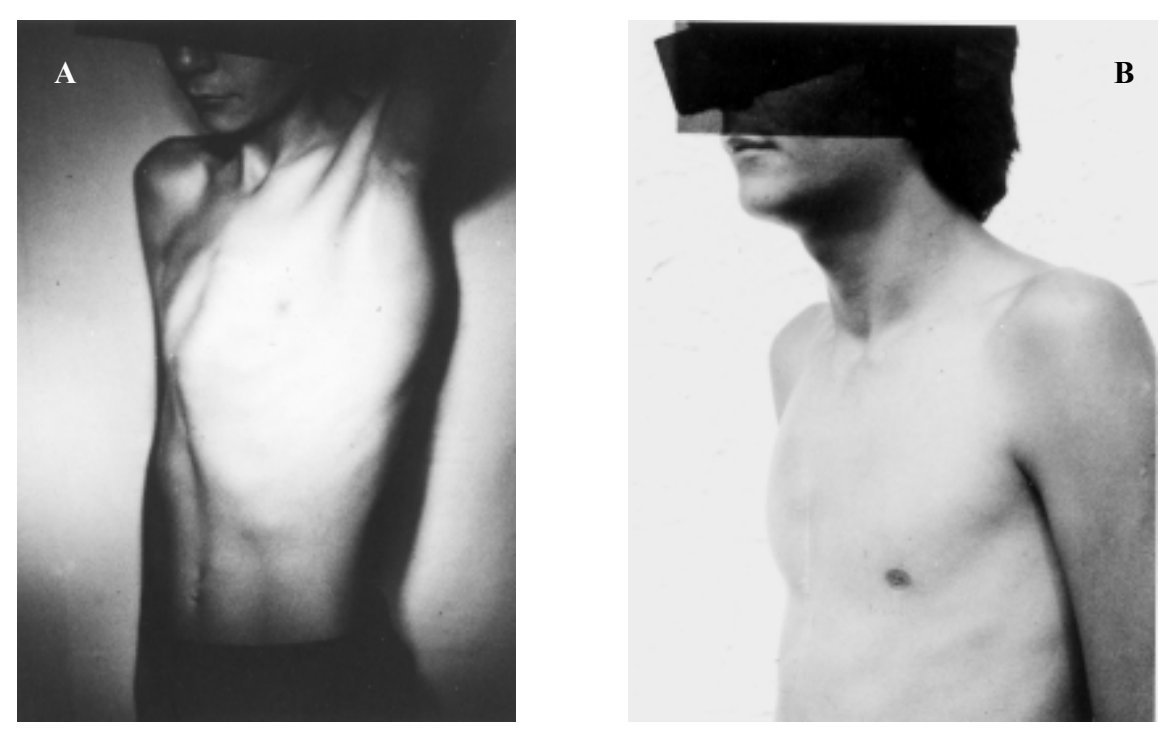

Figura 3 - Pectus Carinatum Inferior Simétrico; A) pré-operatório; B) pós-operatório (3 anos)
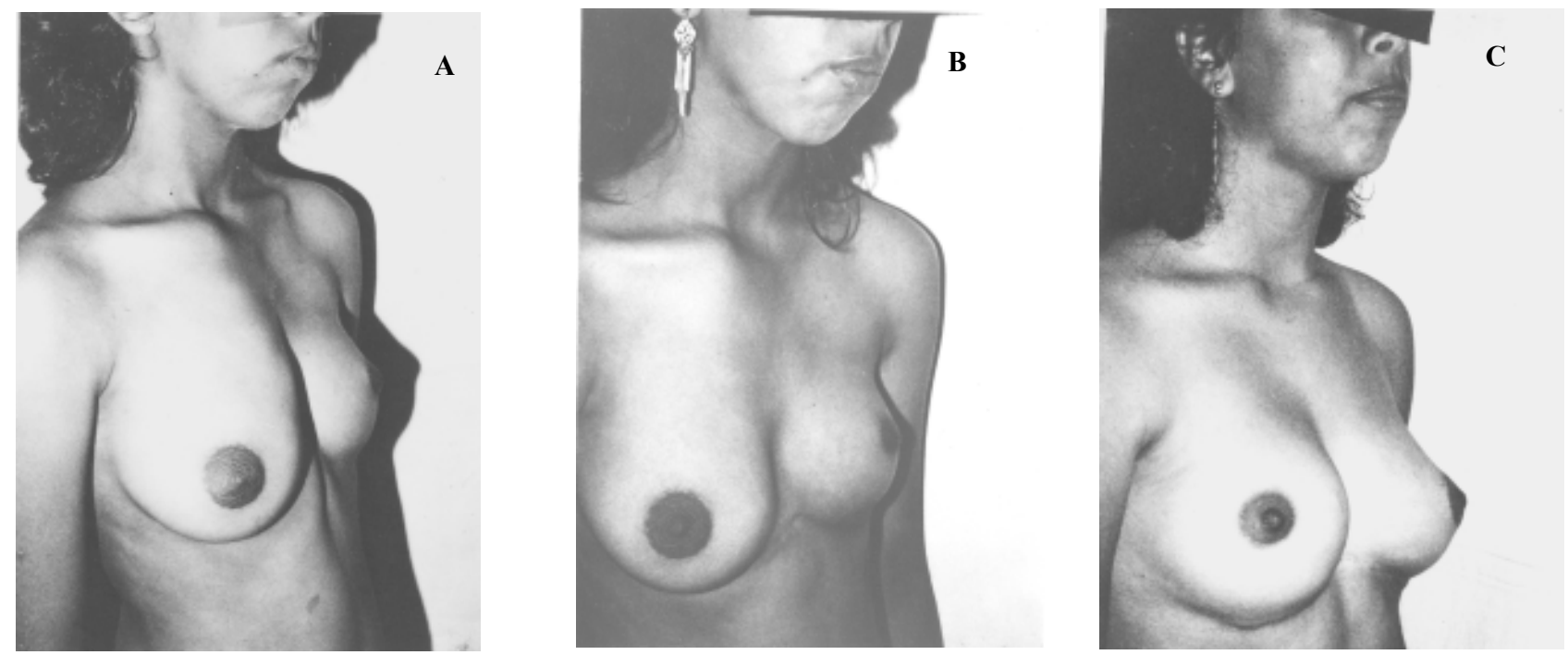

Figura 4 - Pectus Carinatum Lateral associado a assimetria mamária; A) pré-operatório; B) pós-operatório da esternocondroplastia (6 meses); C) pós mastoplastia com prótese de silicone (3 anos)

\section{RESULTADOS}

Os resultados cirúrgicos foram classificados em: a) mau quando houve recidiva da deformidade; b) regular quando o paciente ou familiares não gostaram do resultado alcançado, quando houve cicatriz hipertrófica e quando não se conseguiu dar um contorno completamente satisfatório à parede torácica; e c) bom quando o resultado cirúrgico foi considerado como atingido pela equipe cirúrgica e pelos pacientes e/ou familiares.

Cinqüenta e nove $(95,5 \%)$ dos pacientes portadores de Pectus Excavatum ficaram satisfeitos com o resultado estético obtido. Houve um $(1,6 \%)$ caso de recidiva da deformidade (mau resultado). Tratava-se de paciente com Pectus Excavatum Amplo no qual não foi colocada placa metálica retroesternal e que recusou nova cirurgia. Dois $(3,2 \%)$ não ficaram completamente satisfeitos com o resultado estético obtido, sendo que um deles por ter ocorrido cicatriz hipertrófica (resultado regular).

Cento e dez pacientes $(95,6 \%)$ dos pacientes portadores de Pectus Carinatum Superior e Pectus Carinatum Inferior submetidos à cirurgia ficaram satisfeitos com o resultado estético alcançado (bom resultado). Três $(2,6 \%)$ não 

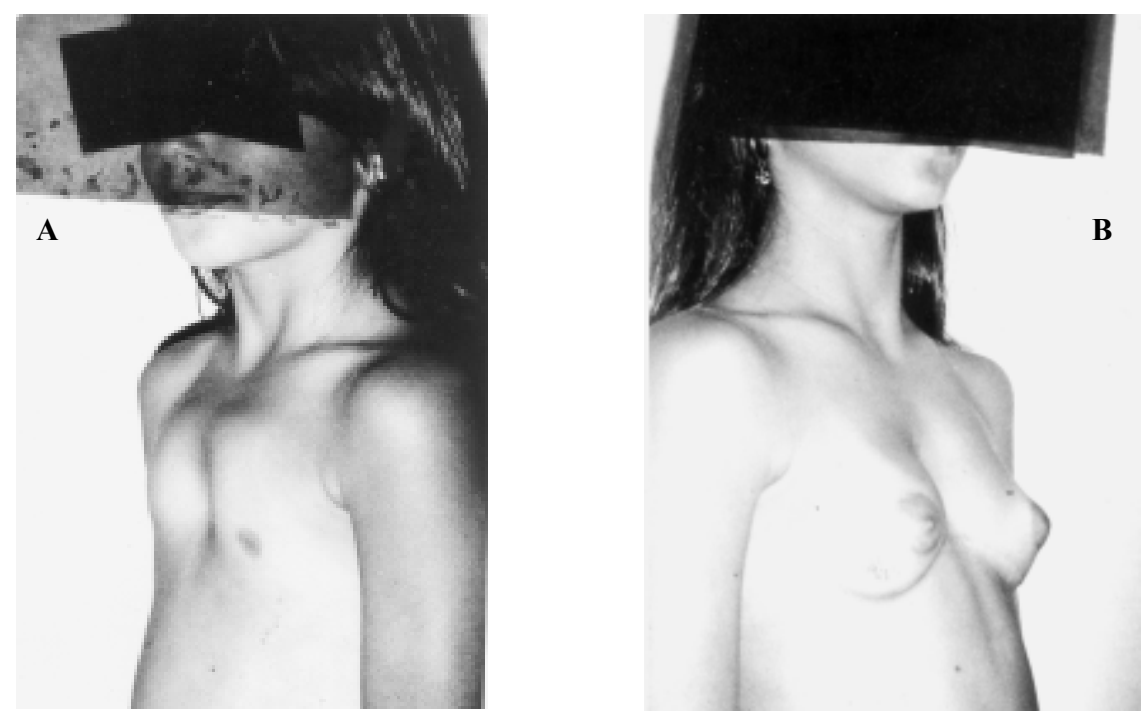

Figura 5 - Pectus Carinatum Superior; A) pré-operatório; B) pós-operatório (5 anos)

Figura 6 - Tipos de "Pectus" e Osteotomia(s) Corretiva(s).

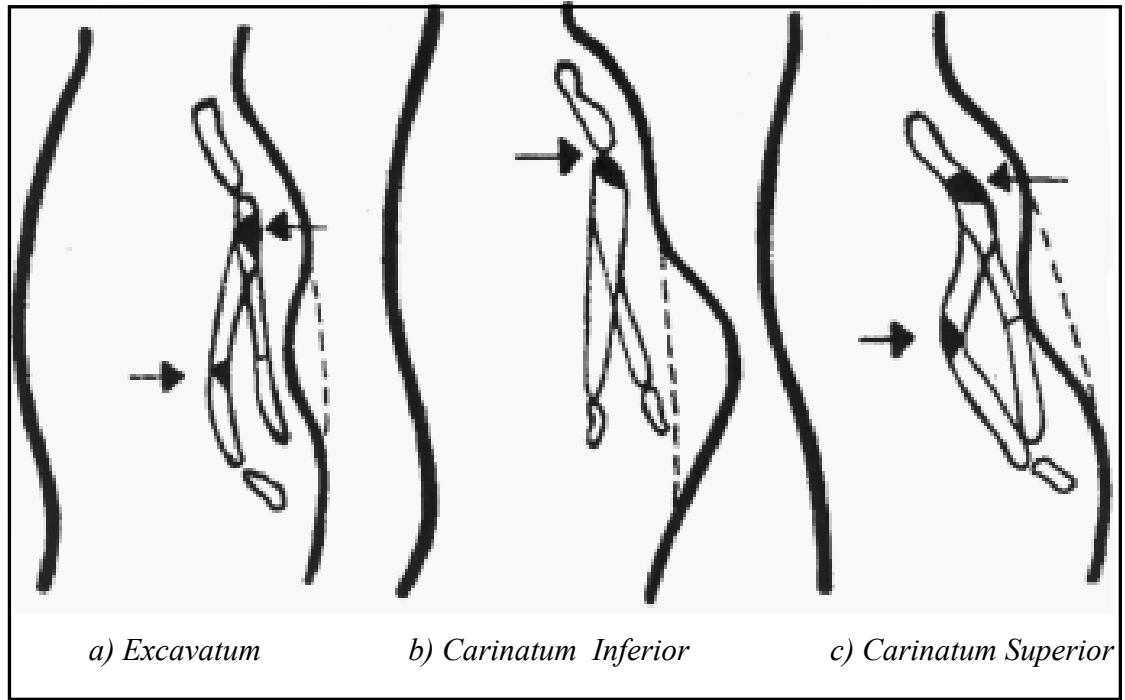

ficaram completamente satisfeitos com o resultado estético (resultado regular), sendo que um destes em virtude de cicatriz hipertrófica. Dois $(1,7 \%)$ pacientes apresentaram depressão esternal no pós-operatório tardio, sendo um de Pectus Carinatum Superior e um de Pectus Carinatum Inferior.

Analisando-se globalmente o resultado foi considerado bom em $175(95,6 \%)$ pacientes. Cinco $(2,7 \%)$ dos pacientes apresentaram resultado regular. Três $(1,6 \%)$ pacientes apresentaram mau resultado, um por recidiva de Pectus Excavatum e dois por depressão esternal após cirurgia para Pectus Carinatum.
Houve uma $(0,5 \%)$ recidiva de Pectus Excavatum o qual recusou a indicação de reoperação (mau resultado).

Complicações ocorreram em $14(7,6 \%)$ pacientes: oito casos $(4,3 \%)$ de seroma no pósoperatório dos primeiros casos, que não mais ocorreram com a drenagem sistemática dos planos submuscular e subcutâneo; um $(0,5 \%)$ de hematoma da parede torácica, a qual situava-se entre o tecido celular subcutâneo e o plano muscular; um $(0,5 \%)$ caso de dor torácica intensa e dois casos $(1,0 \%)$ de cicatriz hipertrófica que foram tratados com ressecção e betaterapia, um $(0,5 \%)$ dos quais com resultado final não completamente satisfatório (Tabela 4). 


\section{DISCUSSÃO}

Os portadores de Pectus Excavatum, na maioria das vezes, são assintomáticos. Por volta da adolescência podem se queixar de dor retroesternal, dispnéia e palpitações de difícil diferenciação com sintomas puramente emocionais. São arredios, introvertidos, eventualmente com complexo de inferioridade, afastados do convívio social e das atividades físicas em que possam expor o tórax. Fadigabilidade fácil, protusão abdominal e má postura com protusão dos ombros para diante e cifose são de ocorrência comum ${ }^{3}$. Arritmias, principalmente atriais, podem ser atribuídas a compressão do coração ou fatores emocionais.

A radiografia do tórax em PA mostra, em alguns casos, desvio do coração para a esquerda. A radiografia do tórax em perfil mostra a depressão esternal em maior ou menor grau, com a articulação manúbrio esternal livre ${ }^{14} \mathrm{e}$ nas crianças e adolescentes as placas cartilaginosas de crescimento esternais estão presentes e individualizadas. A tomografia computadorizada mostra a depressão esternal e o desvio do coração para a esquerda e a deformidade ou compressão cardíaca ${ }^{2}$. As provas de função pulmonar são normais ou levemente alteradas ${ }^{2}$.

Alterações eletrocardiográficas são freqüentemente encontradas e atribuídas a configuração anômala da caixa torácica e ao deslocamento e rotação do coração dentro do hemitórax esquerdo. Podem ser encontradas arritmias, bloqueio do ramo direito, depressão do seguimento ST, desvio do eixo cardíaco, hipertrofia ventricular esquerda, hipertrofia atrial esquerda.

O ecocardiograma demonstra que a compressão do coração pela parede torácica pode deformar o anel mitral ou a câmara ventricular e

Tabela 4 - Deformidades Torácicas Esternocondroplastia.

\begin{tabular}{lcc}
\hline Complicações / 183 & Número & Percentual \\
\hline Seroma & 08 & 4,3 \\
Cicatriz Hipertrófica & 02 & 1,0 \\
Hematoma de Parede & 01 & 0,5 \\
Dor Torácica & 02 & 1,0 \\
Deiscência parcial & 01 & 0,5 \\
\hline Total & 14 & 7,6 \\
\hline
\end{tabular}

produzir o prolapso da válvula mitral nestes pacientes ${ }^{2}$.

O Pectus Carinatum é, na maioria das vezes, progressivo com o crescimento, e acompanhado de pouco ou nenhum sintoma cardio-respiratório. Os sintomas porventura presentes são decorrentes de doença associada ou de perturbações de ordem psicológicas que levam estes pacientes serem introvertidos, desanimados, com complexo de inferioridade, às vezes queixando-se de palpitações, dispnéia, dor torácica, lassidão sintomas que desaparecem com a correção cirúrgica, quando não existe doença associada ${ }^{2,10}$.

Qualquer limitação no trabalho ou atividade ou física ou esportiva deve ser atribuída às alterações de ordem emocional que tal deformidade produz. Estes pacientes evitam freqüentar piscinas, praias e atividades esportivas que exponham o tórax, assumindo posição viciosa de curvar o tórax para diante com aparência "de ombros caídos" para minimizar a visualização do defeito estético ${ }^{10,14}$.

A maioria recomenda a cirurgia para Pectus Excavatum com finalidade estética em virtude do efeito deletério a nível psicológico. A melhora da função cardio-respiratória e do desempenho nas atividades físicas após a cirurgia tem sido atribuída ao bem estar dos pacientes após se verem livres do defeito.

Ravitch ${ }^{13}$ e Lester ${ }^{11}$ preferem operar os portadores de Pectus entre os 2 e 5 anos de idade uma vez que evitar-se-iam os danos psicológicos, a cirurgia seria menos extensa e a cicatriz mais estética. Existe referência a criança operada aos sete meses de idade ${ }^{1}$. Não existe contra indicação cirúrgica, a não ser a presença de doença associada que contra-indique qualquer operação eletiva.

Shamberger ${ }^{2}$ recomenda a cirurgia acima dos cinco anos de idade, Fonkalsrud ${ }^{9}$ entre três e seis anos e Dato ${ }^{3}$ acima dos 10 anos. Nós temos indicado a cirurgia abaixo dos 10 anos quando a deformidade é objetivamente acentuada ou grotesca, uma vez que após os três anos de idade este tipo de deformidades não desaparecerá ${ }^{10}$. A maioria dos nossos casos cirúrgicos $(97,5 \%)$ foram operados após os 10 anos, época que achamos mais apropriada ${ }^{14}$. Acreditamos que, rotineiramente, não devam ser operados os pacientes abaixo dos dez anos para não se lesar as placas cartilaginosas de crescimento e os núcleos de ossificação esternal. 


\section{Técnicas para pectus excavatum}

Ravitch, em $1949^{13}$, relatou a técnica na qual a maioria dos cirurgiões baseou-se para o desenvolvimento do tratamento cirúrgico do Pectus Excavatum. Os princípios da técnica de Ravitch são: ressecção subpericondral das cartilagens envolvidas no defeito, habitualmente da terceira a sétima; separação dos feixes musculares intercostais e pericondriais do esterno bilateralmente; osteotomia esternal transversal posterior a qual é fixada com fio inabsorvível; completa-se a estabilização do esterno com a sobreposição de condrotomias oblíquas efetuadas nas segundas cartilagens costais bilateralmente; e separação do xifóide do esterno.

Welch ${ }^{5}$ recomendava a preservação total das bainhas pericondriais e feixes musculares intercostais para não haver desvascularização esternal e osteotomia esternal anterior com fixação com fio de aço sem suporte esternal. Posteriormente Shamberger e Welch ${ }^{2}$, passaram a utilizar fio de Steimann intraesternal nas crianças acima de 10 anos e, em portadores da Síndrome de Marfan.

Haller ${ }^{14}$ realizaram a condrectomia subpericondral, osteomia posterior e fixação tripodal do esterno e das segundas cartilagens costais após condrotomias oblíquas.

Witz ${ }^{15}$ realizaram condrotomias frontais oblíquas de todas as cartilagens, sem osteotomia, para correção do Pectus Excavatum e Carinatum. Humprheys e Jaretzki ${ }^{16}$ realizam condrotomias e osteotomia esternal.

Judet ${ }^{17}$ e Jung ${ }^{18}$ preconizaram a cirurgia de inversão esternal a qual foi difundida por Wada ${ }^{19}$, que consiste na secção bilateral das cartilagens com secção e inversão esternal. Tal técnica tem elevado índice de infecção e necrose esternal por desvacularização do esterno ${ }^{20}$. Taguschi ${ }^{21}$ recomenda a preservação de ambas as artérias mamárias internas para se evitar esta grave complicação.

Marks ${ }^{22}$ descreveu o implante de silicone abaixo do tecido celular subcutâneo para preencher a depressão. Posteriormente, outros autores publicaram sua experiência com a técnica, salientando a ocorrência de extrusão do molde, recomendando que o implante seja feito no plano submuscular. Rose ${ }^{23}$ relata caso de implante único de silicone para correção da hipoplasia mamária bilateral e Pectus Excavatum. Esta técnica não aumenta o diâmetro da caixa torácica nem diminui a compressão sobre o coração.
Mais recentemente, Marks ${ }^{22}$ utilizou moldagem pré-operatória da depressão do Pectus Excavatum com alginato odontológico e colocou implante de silicone sólido através de incisão com 5 $\mathrm{cm}$ na face ântero-lateral do hemitórax direito. Evitase, desta maneira a incisão sobre a linha média, região onde a ocorrência de cicatriz hipertrófica é maior . A prótese moldada, além do silicone apresenta na sua face interna banda de dacron para promover a sua fixação a parede torácica.

Devido à recidiva e a preocupação de se dar maior rigidez a caixa torácica, vários autores utilizam suporte retroesternal com vários materiais: marlex retroesternal ${ }^{4}$, placa metálica retroesternal ${ }^{9}$, fio de Kirchner retroesternal ${ }^{24}$, dacron vascular ${ }^{11}$, enxerto vascularizado de costela, e prótese metálica em asa de gaivota ${ }^{3}$.

Wooler ${ }^{6}$, em 1969, publicou um método divulgado pelo professor Jesse Teixeira ${ }^{26}$, que consiste na ressecção subpericondral das cartilagens envolvidas, dissecção retroesternal, divisão dos feixes musculares intercostais e do pericôndrio do esterno, osteotomia esternal posterior e pericardioplastia do pericárdio redundante.

Com o desenvolvimento de cirurgia minimamente invasiva, Nuss $^{27}$ baseado nas evidências de que as crianças tem tórax mole e maleável; na remodelação do tórax que ocorrem em adultos que desenvolvem enfisema; no desenvolvimento de pectus carinatum nas crianças portadores de asma brônquica e no uso de órteses pelos ortopedistas e ortodontistas para correção, respectivamente de deformidades da coluna vertebral e dos dentes, desenvolveu técnica em que utiliza uma placa arcada que é introduzida na face lateral de um hemitórax através de incisão com $5 \mathrm{~cm}$ e tracionada através do mediastino anterior por pinça introduzida no outro hemitórax com a parte arcada para cima. A placa é rodada de modo a parte arcada ficar para baixo e é fixada nas costelas. A placa permanece por 2 anos. Apresentou 13,3\% de maus resultados e índice de complicações de $25,0 \%{ }^{27}$.

Posteriormente, $\mathrm{Hebra}^{28}$, utilizou toracoscopia para visualização da passagem da placa pelo mediastino. O índice de complicações foi elevado $(21,0 \%)$, inclusive um caso de perfuração do átrio direito, ventrículo direito e lesão da válvula tricúspide.

Kobayashi ${ }^{29}$ realizou a correção de Pectus Excavatum e Pectus Carinatum assistida por 
videoendoscópio introduzido através de incisão de 4 $\mathrm{cm}$. na altura da base do processo xifóide que também é utilizada para dissecção do tecido celular subcutâneo e exposição do esterno e cartilagens envolvidas. Uma incisão com $3 \mathrm{~mm}$ em cada hemitórax são feitas para introdução de descoladores, tesoura e cautério. Executa a ressecção parcial das cartilagens envolvidas, osteotomia esternal transversal e colocação de placa metálica retroesternal. Em 8 casos operados o tempo cirúrgico variou de 5,4 a 10 horas com média de 6,7 horas.

\section{Técnica para pectus carinatum}

Lester ${ }^{11}$, em 1953, operou o primeiro caso utilizando a ressecção subperiostal do corpo do esterno e, posteriormente também das cartilagens costais envolvidas. Chin ${ }^{30}$ procedeu a ressecção subpericondral das cartilagens envolvidas e xifoesternopexia. Welch ${ }^{5}$ ressecou as cartilagens envolvidas, normalmente da segunda a sétima, realizando uma ou várias osteotomias e fixando com fio inabsorvível a nova moldagem do esterno. Robiseck ${ }^{31}$ preconiza a ressecção subpericondral das cartilagens envolvidas no Pectus Clássico, Lateral e Pouter Pigeon, com a ressalva que, habitualmente neste, podem ser necessárias duas ou mais osteotomias para se atingir resultado estético favorável. Tjan ${ }^{32}$ em 111 pacientes obteve $98,2 \%$ de bons resultados utilizando a ressecção subpericondral, osteotomia esternal, colocação de duas placas paralelas verticais em cada hemitórax na borda costal de onde foram retirada as cartilagens e que tem a função de longarinas, e pino transesternal que se apóia nas 2 placas verticais ${ }^{32}$.

A partir da Técnica de Ravitch ${ }^{13}$, temos desenvolvido, desde $1976{ }^{33}$, técnica única com preocupação estética e funcional para as várias formas de Pectus com resultados estéticos excelentes em $95,6 \%$ dos pacientes. Os resultados obtidos a nosso ver são decorrentes de não seccionarmos as bandas musculocartilaginosas e as preguearmos de uma forma tensa de modo a dar firmeza à parede torácica; de procedermos as osteotomias anteriores sem grandes descolamentos retroesternais; de as fixarmos com fio de aço e ao cuidado com a sutura da pele.

No tratamento do Pectus Carinatum, eventualmente tem sido utilizada como tratamento único a fisioterapia, porém há, praticamente unanimidade em afirmar-se que não há resultado com este tipo de tratamento ${ }^{15,22,34,35}$. A melhora observada seria a daqueles defeitos pequenos que seriam corrigidos com o crescimento e passíveis apenas de observação.

Há referências ao tratamento ortopédico com o uso de aparelhos e cintos, com o objetivo de cumprir o tórax e, portanto, modelar o defeito. Entre nós Haje e Raimundo ${ }^{34}$ fazem uso de aparelho denominado "Compressor Dinâmico do Tórax", que faz a compressão seletiva sobre o esterno, utilizando como contra-força a coluna vertebral. A sua utilização é baseada na capacidade de remodelação óssea conforme as forças a que o osso é submetido (Lei de Julius Wolff). Tal aparelho tem a vantagem sobre as faixas e cintos, os quais fazem compressão em toda a caixa torácica com prejuízos óbvios para a ventilação. Seu inconveniente é a necessidade de uso diário e prolongado. O paciente deve usar o aparelho 23 horas por dia. Retira o aparelho apenas em três sessões diárias de fisioterapia. Em publicações de $1992{ }^{36}$ cita controle em um ano, porém não faz menção a duração de tratamento necessária para se obter a cura definitiva. Em trabalho anterior cita caso com duração além de dois anos ${ }^{37}$.

No Pectus Carinatum Clássico e Lateral obteve resultados parciais em $36,3 \%$ e melhoria significativa em $63,7 \%$. Não faz menção à cura nem a recidiva da deformidade. Nos três casos de Pouter Pigeon obteve melhora parcial em dois casos e um caso de melhora significativa. Nos parece, portanto que não é convincente para uso generalizado, estando indicado nas crianças e adolescentes em forma medianas e discretas de Pectus Carinatum e Lateral, sabendo de antemão a resistência das crianças e adolescentes a este tipo de tratamento.

Nas formas discretas e medianas temos recomendado aos pacientes que tenham vida normal com atividades esportivas variadas, principalmente natação.

Fonkalsrud et al. ${ }^{9}$ relatam que $90 \%$ das crianças com sintomas respiratórios no pré-operatório, melhoraram após a correção de Pectus Excavatum. É conhecida a melhora na atividade física e no comportamento das crianças e adolescentes após cirurgia ${ }^{4}$.

Ravitch ${ }^{1}$ relata $54 \%$ de resultados satisfatórios em 20 anos. Humpreys e Jaretzki ${ }^{38}$ relatam 64\%.

Com o seguimento médio de cinco anos obtiveram resultados satisfatórios: Hawkins ${ }^{39}(81 \%)$ e Prevot ${ }^{35}(90 \%)$. 
Cita-se como fatores preditivos de maus resultados: peso elevado por ocasião da cirurgia, defeito severo e associação com Síndrome de Marfan ${ }^{2,31}$.

$\mathrm{Na}$ nossa série não tivemos complicações graves. O seroma não tem ocorrido em virtude da drenagem sistemática do TCS e do plano submuscular. Os dois pacientes que apresentaram cicatriz hipertrófica se beneficiaram com a ressecção e betaterapia no sexto dia.

Várias complicações têm sido relatadas: seroma ${ }^{2,9,29,35}$, hematoma ${ }^{10}$, infecção da ferida ${ }^{3,10}$, pneumotórax ${ }^{2,3,6,9}$, hemoptise ${ }^{12}$, hemopericárdio ${ }^{2,12}$, tamponamento cardíaco ${ }^{3}$, necrose de pele ${ }^{40}$, quelóide ${ }^{10,41}$, necrose esternal ${ }^{39}$, atelectasia ${ }^{9,4}$ e recidiva. O índice de mortalidade está entre 0 e $0,5 \%$. ${ }^{33}$.
Levando em consideração o excelente resultado estético alcançado $(95,6 \%)$, o baixo índice de recidiva ou inversão da deformidade nesta série $(1,6 \%)$, a não ocorrência de complicações sérias e as perturbações de ordem psicológica e posturais que estão presentes em muitos portadores destas deformidades, a indicação cirúrgica se faz, quando a deformidade torácica seja acentuada e o paciente e/ou familiares a deseje. Os portadores de Pectus Carinatum Simétricos ou Laterais discretos ou medianos em pacientes ainda em fase de crescimento são encaminhados para tratamento ortopédico para modelar o tórax. Salienta-se que são operados apenas cerca de $10 \%$ dos pacientes a nós referidos.

\begin{abstract}
Background: The authors present the results obtained with one technique for Pectus Carinatum and Pectus Excavatum treatment. Methods: From 1976 to 2000, 183 patients with chest wall deformities underwent surgery at the Hospital Universitario Cajuru of the Catholic University of Parana. Nighty eight were pectus carinatum (70 symmetric pectus carinatum, 18 lateral pectus carinatum to the right and 10 lateral pectus excavatum to the left), 62 were pectus excavatum (57 symmetric pectus excavatum, four lateral pectus excavatum to the right and one lateral pectus excavatum to the left), 17 were pouter pigeon, one was pectus carinatum with pectus excavatum, four were inferior costal protusions and one costal depression. The indication was exclusively aesthetical in $182(99,4 \%)$ of the patients. For both pectus carinatum and pectus excavatum only one technique was used: inframammary transversal incision, subperichondral ressection of all cartilages involved in the deformity, minimal retrosternal dissection, anterior sternal osteotomy, fastening of the sternal osteotomy with a steel wire. A retrosternal plate can be used in selected Pectus Excavatum cases, folding of the perichondrial bundles to give more rigidity to the thoracic wall and to help keep the sternum in its position, drainage of the subcutaneous cellular tissue and of the submuscular space, intradermal suture of the skin.Results: A good and / or excellent aesthetic result was obtained in 175 (95,6\%) of the patients. Complication occurred in 14 (7,6\%) patients: eight (4,5\%) seroma cases, one (0,5\%) hematoma of the chest wall, two (91,0\%) cases of severe chest pain in the postoperative period; one (0,5\%) case of dehiscence of skin suture and two cases (1,0\%) of hyperthrophic scar, which were treated with ressection and betatherapy. Conclusion: The aesthetic results obtained allow us indicate the sternochondroplasty for treatment of Pectus Carinatum and Pectus Excavatum.
\end{abstract}

Key Words: Funnel chest; Abdormalities; Surgical procedures, operative.

\section{REFERÊNCIAS}

1. Ravitch MM - "Pectus excavatum”. In Ravitch MMCongenital deformites of the chest wall and their operative correction. Philadelphia. WB Saunders, 1997, pp. 206-232.
2. Shamberger RC - "Chest wall deformities". In Thomas C. Schields (ed)-General thoracic surgery. $4^{\text {th }}$ Edition. Willians \& Wilkins, 1994, pp. 529-557.

3. Dato GMA, De Paulis R, Dato AA, et al.-Correction of pectuc excavatum with a self- retaing seagull wing prosthesis. Long-term follow-up. Chest, 1955, 107:303-306. 
4. Robicsek F, Fokin A - Surgical correction of pectus excavatum and carinatum. J Cardiovasc Surg, 1999, 40(5):725-731.

5. Welch KJ, Vos A - Surgical correction of pectus carinatum (pigeon breast). J Pediatr Surg, 1973, 8(5):659667.

6. Wooler GH, Mashnour YA, Garcia JB, et al. - Pectus excavatum. Thorax, 1969, 24(5): 557-562.

7. Clark JG, Greenville-Mathers R - Pectus Excavatum. Chest, 1962, 56:202-205.

8. Coelho MS, Guilherme EV, Kume MK, et al-Incidência de deformidades torácicas entre escolares de Curitiba. J Pneumol, 1982, 8 (Supl), 175.

9. Fonkalsrud EW, Salman T, Guo W, et al. - Repair of pectus deformities with sternal suport. J Thorac Cardiovasc Surg, 1994, 107(1):37-42.

10. Coelho MS, Von Bathen LC, Guzzi A, et al. - "Pectus excavatum" / "Pectus Carinatum:" resultados do tratamento cirúrgico. Rev Bras Ortop, 1988, 23(5):120124.

11. Lester CW - Surgical of protusion deformities of the sternum and costal cartilages (pectus carinatum, pigeon breast). Ann Surg, 1961, 153:441-446.

12. Currarino G, Silverman F - Premature obliteration of sternal suture and pigeon-breast deformity. Radiology, 1958, 70:532-540.

13. Ravitch MM - The operative treatment of Pectus excavatum. Ann Surg, 1949, 129:429-44.

14. Haller JA, Shermeta DW, Tepas JJ, et al. - Corrections of pectus carinatum whithout prostheses or splints: objective measuremet of severity and management of asymmetrical deformities. Ann Thorac Surg, 1978, 26(1):73-79.

15. Witz JP, Morand G, Wihlm JM - Thorax en entonnoir, thorax en carene. Traitement par sterno-chondroplastie. A propos de 43 interventions. Ann Chir Thorac Cardiovasc, 1976, 15(2):157-160.

16. Humphreys GH, Jaretzki A - Operative corretion of pectus excavatum. Pediat Surg, 1974, 9(6):899-909.

17. Judet J, Judet $\mathrm{R}$ - Thorax en entonnoir. Un procedé operatoire. Rev Orthop 1954, 40:248.

18. Jung A - Le traintement du thorax en entonnoir par le "retournement pédiculé" de la cuvette sternochondrale. Mém Acad Chir, 1956, 82:242.

19. Wada J, Ikeda I, Ishida T, et al. - Results of 271 funnel chest operations. Ann Thorac Surg, 1970, 10(6): 526532.

20. Doty BD, Hawkins JA - A turnover operation for pectus excavatum at time of corretion of intracardiac defects. J Thorac Cardiovasc Surg, 1983, 86(5):787-790.

21. Taguchi K, Mochizuki T, Nakagaki M, et al. - A new plastic operation for pectus excavatum: sternal turnover surgical procedure with preserved internal mammary vessels. Chest, 1975, 67(5): 606-608.
22. Marks MW, Iacobucci J - Reconstruction of congenital chest wall deformities using solid silicone onlay prostheses. Chest Surg Clin North Am, 2000, 10(2): 341-355.

23. Rose EH, Lavey EB - Correction of bilateral breast hypoplasia and pectus excavatum with single-unit customized silicone implant. Plast Reconst Surg, 1983, 72(2): 234-236.

24. Bentz ML, Futrell JW - Improved chest wall fixation for correction of pectus excavatum. Br J Plast Surg, 1992, 45(5): 367-370.

25. Hayashi A, Maruyama Y - Vascularized rib strut technique for repair of pectus excavatum. Ann Thorac Surg, 1992, 53(2): 346-348.

26. Teixeira JP, Teixeira Filho PJ - Operative correction of pectus excavatum using the right ventricle as a sternal support. J Am Coll Surg, 1995, 180(3): 346-349.

27. Nuss D, Kelly RE, Croitoru DP, et al. - A 10-year review of a minimally invasive technique for the correction of pectus excavatum. J Pediatr Surg, 1998, 33(4): 545-552.

28. Hebra A - Minimally invasive pectus surgery. Chest Surg Clin North Am, 2000, 10(2): 329-339.

29. Kobayashi S, Yoza S, Komuro Y, et al. - Correction of pectus excavatum and pectus carinatum assisted by the endoscope. Plast Reconstr Surg, 1995, 99(4):10371045.

30. Chin EF - Surgery of funnel chest and congenital sternal prominence. Br J Surg, 1957, 44: 360-376.

31. Robicsek F - Surgical treatment of pectus carinatum. Chest Surg Clin North Am, 2000, 10(2): 357-375.

32. Tjan TD, Semik M, Rotering H, et al. - Pectus excavatum: special surgical technique, perioperative management and long-term results. J Cardiovasc Surg, 1999, 40(2): 289-297.

33. Coelho MS, Pizarro LV, Santos AG - Pectus carinatum: tratamento cirúrgico. Rev Med Paraná, 1981, 41(1/2): 9-13.

34. Haje AS, Raymundo JLP - Considerações sobre deformidades da parte torácica anterior e apresentação de tratamento conservador para as formas de protusão. Rev Bras Ortop, 1979, 14(5): 164-177.

35. Prévot J - Treatment of sternocostal wall malformations of the child. A series of 210 surgical corretions since 1975. Eur J Pediatr Surg, 1994, 4(3):131-136.

36. Haje SA, Bowen JR - Preliminary results of orthotic treatment of pectus deformities in children and adolescents. J Pediatr Orthop, 1992, 12(6):795-800.

37. Haje SA, Antunes EJ, Raymundo JL, et al. - Pectus Carinatum: enfoque atual. Rev Bras Ortop,1988, 23(9):257-264.

38. Humphreys HG, Jaretzki A - Pectus excavatum. Late results with and without operation. J Thorac Cardiovasc Surg, 1980, 80(5): 685-695. 
39. Hawkins JA, Ehrenhaft JL, Doty DB - Repair of pectus excavatum by sternal eversion. Ann Thorac Surg, 1984, 38(4): 368-373.

40. Wesselhoeft CW, De Luca FG - A simplified approach to the repair of pediatric pectus deformities. Ann Thorac Surg, 1982, 34(6): 640-646.

41. Jones WG, Hoffman L, Devereux RB, et al. - Staged aproach to combined repair of pectus excavatum and lesions of the heart. Ann Thorac Surg, 1994, 57(1):212-214.
Endereço para correspondência:

Dr. Marlos de Souza Coelho

Clínica do Tórax

Av. Comendador Franco (Av. das Torres), 2429

Guabirotuba - CEP 81520-000 - Curitiba-PR

Fone (0xx41) 266-3500 FAX(0xx41) 266-4349

Site: www.marloscoelho.com.br

E.mail: clinicadotorax@marloscoelho.com.br 